\title{
Bending Crystals: Emergence of Fractal Dislocation Structures
}

\author{
Yong S. Chen, Woosong Choi, Stefanos Papanikolaou, and James P. Sethna \\ Laboratory of Atomic and Solid State Physics (LASSP), Clark Hall, Cornell University, Ithaca, New York 14853-2501, USA
}

(Received 8 March 2010; published 1 September 2010)

\begin{abstract}
We provide a minimal continuum model for mesoscale plasticity, explaining the cellular dislocation structures observed in deformed crystals. Our dislocation density tensor evolves from random, smooth initial conditions to form self-similar structures strikingly similar to those seen experimentallyreproducing both the fractal morphologies and some features of the scaling of cell sizes and misorientations analyzed experimentally. Our model provides a framework for understanding emergent dislocation structures on the mesoscale, a bridge across a computationally demanding mesoscale gap in the multiscale modeling program, and a new example of self-similar structure formation in nonequilibrium systems.
\end{abstract}

DOI: 10.1103/PhysRevLett.105.105501

Structural engineering materials have a bewildering variety of microstructures, which are often controlled by deformation and annealing during the formation process. An imposed distortion generates a complex morphology even for a single crystal of a pure material - polycrystalline grains form at high temperature where dislocation climb allows for polygonization, cell structures form at low temperatures when climb is forbidden. Cell walls [Figs. 1(c) and 1(d)] are distinct from grain boundaries in that they have smaller misorientations, different origins, are morphologically fuzzier, and the cells refine (get smaller) under shear. Experiments differ in characterizing the cell structures; some show convincing evidence of fractality [1-3] with structure on all length scales [Fig. 1(c)], while others show structures with a single characteristic scale setting their cell size and cell wall misorientation distributions [4-6] [Fig. 1(d)].

Dislocation avalanches [7], size-dependent hardness (smaller is stronger) [8], and cellular structures [1,5] all emerge from collective dislocation interactions on the micron scale. We expect that these mesoscale phenomena should be captured by an appropriate continuum theory of dislocation dynamics. Computationally, such a theory is crucial for multiscale modeling, as atomistic and discrete dislocation simulations are challenging on these scales of length and strain. Here we present a minimal model for cellular structures, which eventually can be extended to include the pinning and entanglement needed for avalanches and hardness, and the slip systems and statistically stored dislocations needed for realistic descriptions of texture evolution and cross slip [9]. Our model gives the elegant, continuum explanation for the formation and evolution of cellular dislocation structures. It exhibits both the experimentally observed fractal structures and scaling collapses hitherto thought incompatible. Finally, it provides the fundamental distinction between cell walls and grain boundaries; cell walls are intrinsically branched in a fractal fashion.
PACS numbers: 61.72.Bb, 05.45.Df, 05.45.Pq, 61.72.Lk

Within a continuum theory of dislocation dynamics $[10,11]$, incorporating only elastic self-interactions with a minimally modified gradient dynamics, we study the relaxation of a smoothly deformed crystal and its subsequent evolution under external strain [Figs. 1(a) and 1(b)]. When climb is allowed, we find the distortion neatly evolves into a stress-free collection of grain boundaries. When climb is forbidden, cell wall structures evolve with power-law correlations and self-similarity-providing a clear morpho-

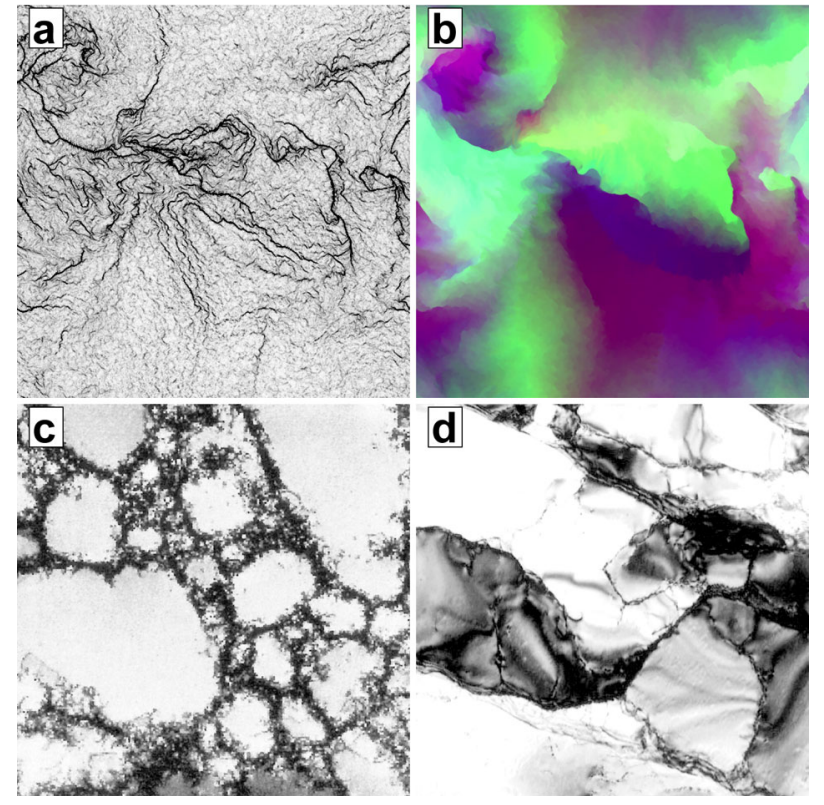

FIG. 1 (color online). Theoretical and experimental dislocation fractal morphologies. Top: Simulated fractal cell wall pattern after uniaxial strain of $\epsilon_{z z}=4 \beta_{0}$. (a) Dislocation density plot; (b) local orientation map. Bottom: TEM micrographs taken from (c) a $\mathrm{Cu}$ single crystal [1] after [100] tensile deformation to a stress of $75.6 \mathrm{MPa}$ and (d) an Al single crystal following compression to $\epsilon=0.6$ [5], respectively. Gray scales have been adjusted to facilitate visual comparisons. Note the striking morphological similarity between theory and experiment. 


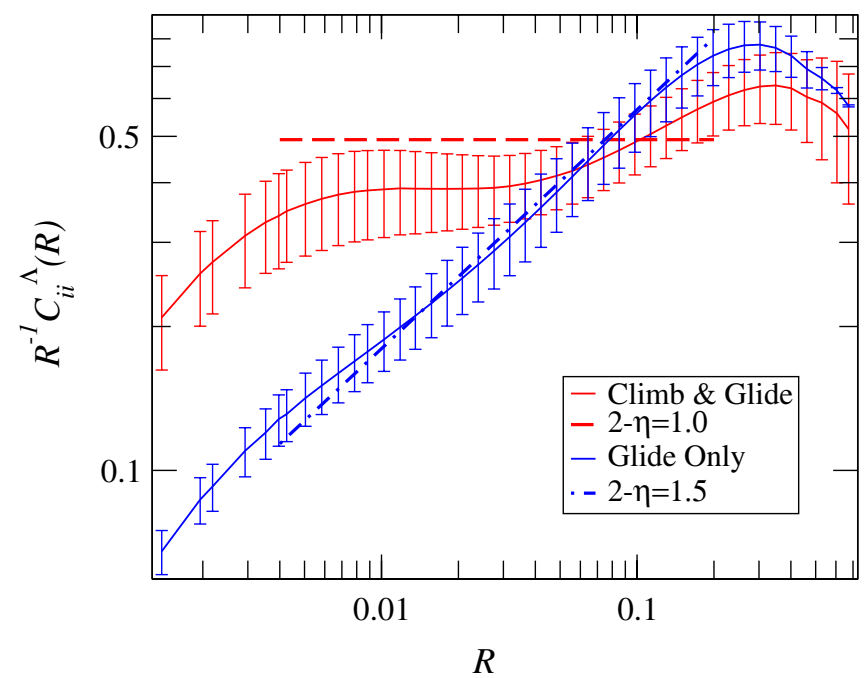

FIG. 2 (color online). Scaling of the correlation function $\left(1024^{2}\right.$ simulations). The trace of the orientation-orientation correlation function $C_{i j}^{\Lambda}(R)=\left\langle\left(\Lambda_{i}(\mathbf{x})-\Lambda_{i}(\mathbf{x}+\mathbf{r})\right)\left(\Lambda_{j}(\mathbf{x})-\right.\right.$ $\left.\left.\Lambda_{j}(\mathbf{x}+\mathbf{r})\right)\right\rangle$ is averaged over all pairs of points at distance $|\mathbf{r}|=$ $R$. Notice that the simulation allowing climb has $C_{i i}^{\Lambda}(R) \sim R$ as expected for nonfractal grain boundaries. Notice that the glideonly simulations show $C_{i i}^{\Lambda}(R) \sim R^{2-\eta}$ with $\eta \approx 0.5$, indicating a fractal, self-similar cell structure, albeit cut off by lattice and system size effects.

logical distinction between cell walls and grain boundaries, a tangible model for the experimentally observed fractal structures [1-3], and an alternative to those that predict microstructure without a wide range of scales [12]. The resulting morphology, however, is self-similar only after rescaling both space and amplitude. Performing the experimentalist's analysis of the misorientation and cell-size distributions [4-6] yields good agreement with the observed scaling form (albeit with significantly different scaling functions and exponents). By directly exhibiting key features of the emergent experimental behavior in a continuum, deterministic dislocation density theory, our simulations pose a challenge to theories based on stochasticity in the continuum laws $[3,13]$ or in the splittings and rotations of the macroscopic cells $[14,15]$. Can these stochastic theories describe our chaotic dynamics after coarse graining?

Our order parameter is the plastic distortion tensor $\beta^{P}$. Together with the resulting elastic distortion $\beta^{E}$ derivable from $\beta^{P}$ via the long-range fields of the dislocations [11], $\beta^{P}$ both gives the deformation $\mathbf{u}$ of the material (through $\partial_{i} u_{j}=\beta_{i j}^{E}+\beta_{i j}^{P}$ ) and gives a three-index variant of the Nye dislocation density tensor [16] $\rho_{i j k}(\mathbf{x})=\partial_{j} \beta_{i k}^{P}-$ $\partial_{i} \beta_{j k}^{P}$ (defining the flux of dislocations with Burgers vector along the coordinate axis $\hat{\mathbf{e}}_{k}$ through the infinitesimal surface element along $\hat{\mathbf{e}}_{i}$ and $\hat{\mathbf{e}}_{j}$ ). $\beta^{P}$ thus fully specifies the dislocation wall morphologies, the crystal rotation (the Rodrigues vector $\boldsymbol{\Lambda}$ giving the axis and angle of rotation), and the stress field $\sigma$ [the external load plus the long-range
TABLE I. Critical exponents measured for different correlation functions. GO: glide only; CG: climb and glide; ST:scaling theory [18].

\begin{tabular}{lccc}
\hline \hline Correlation functions & GO & CG & ST \\
\hline$C_{i i}^{\Lambda}(\mathbf{r})=\left\langle\sum_{i}\left[\Lambda_{i}(\mathbf{r})-\Lambda_{i}(0)\right]^{2}\right\rangle$ & $1.5 \pm 0.1$ & $1.1 \pm 0.1$ & $2-\eta$ \\
$C^{\rho}(\mathbf{r})=\left\langle\left[\rho_{i j}(0) \rho_{i j}(\mathbf{r})\right]\right\rangle$ & $0.4 \pm 0.1$ & $0.9 \pm 0.3$ & $\eta$ \\
\hline \hline
\end{tabular}

stresses from the dislocations, given by a kernel $[11,17]$ $\left.\sigma_{i j}(\mathbf{r})=\sigma_{i j}^{\text {ext }}+\int K_{i j k l}\left(\mathbf{r}-\mathbf{r}^{\prime}\right) \rho_{k l}\left(\mathbf{r}^{\prime}\right) d \mathbf{r}^{\prime}\right]$.

Following Roy and Acharya [10], we assume the flow of $\rho_{i j k}(\mathbf{x})$ is characterized by a single velocity $\mathbf{v}(\mathbf{x})$. Allowing both climb and glide, we can take the velocity $\mathbf{v}$ to be proportional to the Peach-Kohler force $\mathcal{F}$ on the entire population of dislocations times a mobility $D(|\rho|) v_{a}=$ $D(|\rho|) \mathcal{F}_{a}=D(|\rho|) \rho_{\text {ast }} \sigma_{\text {st }}$, where $\sigma$ is the stress; we then define $\partial \beta_{i j}^{P} / \partial t=J_{i j}=v_{a} \rho_{a i j}$. (This provides the same equation of motion derived later by Limkumnerd and Sethna [11].) To remove dislocation climb (mass transport via frozen-out vacancy diffusion), we must set the trace of the volume change $J_{i i}=0$, suggesting a dynamics which moves only the traceless portion of the dislocation density:

$$
\frac{\partial \beta_{i j}^{P}}{\partial t}=J_{i j}=v_{a} \rho_{a i j}-\frac{1}{3} \delta_{i j} v_{a} \rho_{a k k} .
$$

In this case, to guarantee that energy monotonically de-

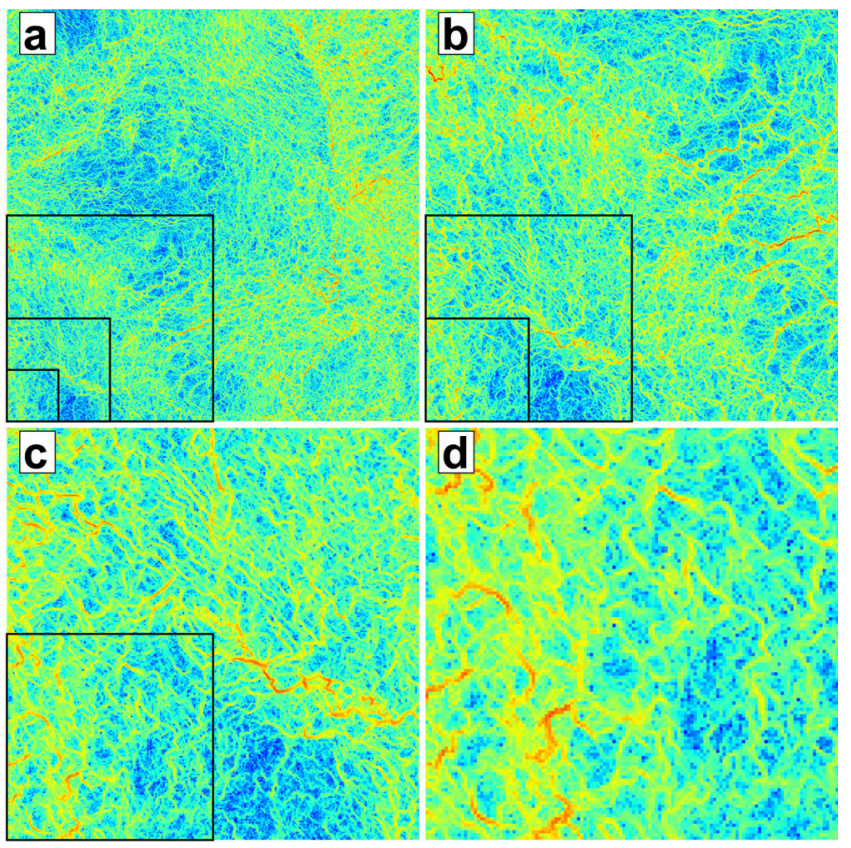

FIG. 3 (color online). Self-similarity in real space. Each frame represents the lower left-hand quarter of the previous frame. Frame (a) is a $1024 \times 1024$ simulation; (b), (c), and (d) are thus of length $L=512,256$, and 128. All are rescaled in amplitude by $\left(L / L_{0}\right)^{-\eta / 2}$ with $\eta=0.5$ (see Fig. 2 and Table I). The scale is logarithmic with a range of almost $10^{7}$. Notice the statistical selfsimilarity. Other regions, when expanded, can show larger differences between scales, reflecting the macroscopic inhomogeneity of the dislocation density. 


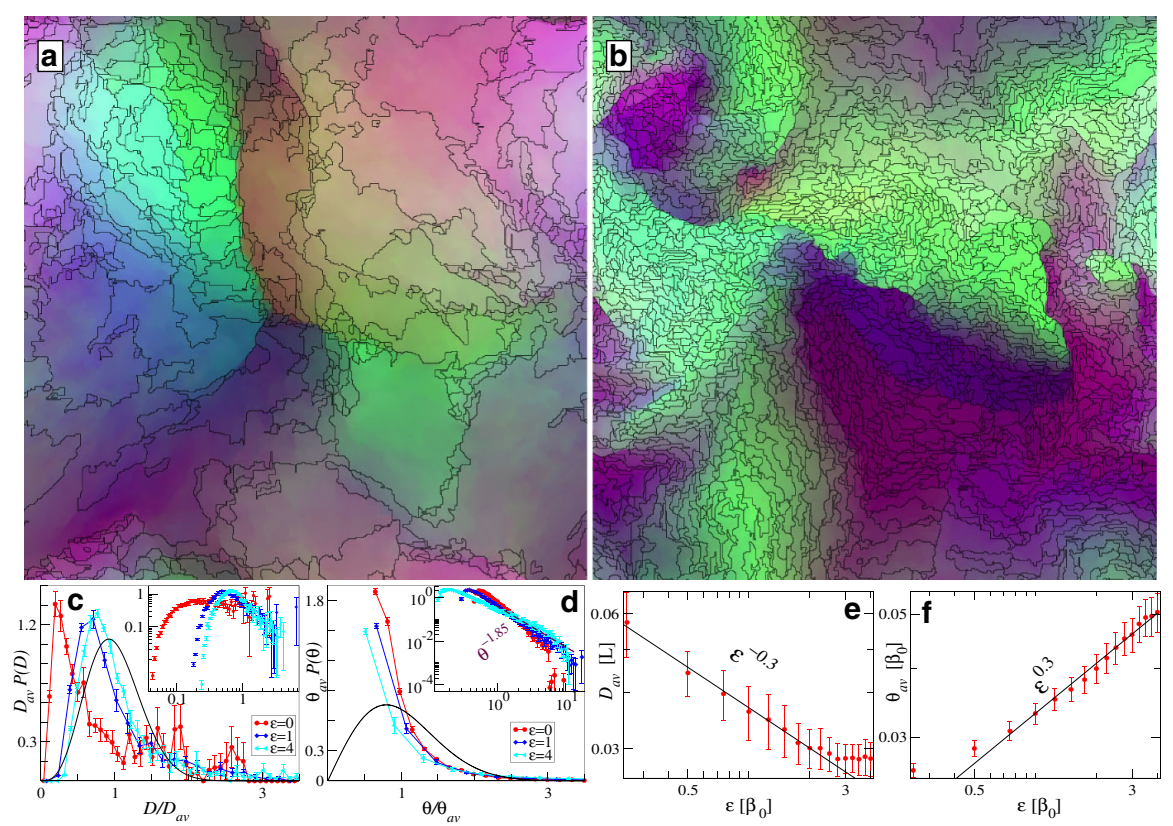

FIG. 4 (color online). Cellular structures under strain: size and misorientation distributions. (a) An unstrained state formed by relaxing a random deformation, decomposed into cells determined by our boundary-pruning method: we systematically remove boundaries in order of their average misorientation angle, and then prune cells based on their perimeter-to-area ratios and misorientation angles (see supplementary movie 4 [21]). Boundaries below a threshold root-mean-square misorientation $\theta_{c}=$ $0.015 \beta_{0}$ are removed. (b) The final state after a strain of $\epsilon_{z z}=4 \beta_{0}$ is applied; notice the cell refinement to shorter length scales. (c) The cell-size distribution (square root of area), scaled by the mean cell size and weighted by the area, at various external strains. (d) The misorientation angle distribution, weighted by cell boundary length, scaled by the mean. For each curve, data start at $\theta_{c}$. This distribution appears to be closer to a power-law (inset) than the experimental distributions (solid curves [4-6]). (e),(f) Mean cell size $D_{\text {av }}$ and misorientation angle $\theta_{\mathrm{av}}$ as functions of external strain. We find these same power laws $D_{\mathrm{av}} \sim \epsilon^{-0.26 \pm 0.14}$ and $\theta_{\mathrm{av}} \sim \epsilon^{0.26 \pm 0.04}$, with errors reflecting over a range of $\theta_{c}$ and for a variety of pruning algorithms and weighting functions. Notice that the product $D_{\text {av }} \theta_{\text {av }}$ is approximately constant, as observed experimentally [4]. The power-law dependence $\epsilon^{0.3}$ is weaker than the powers $\epsilon^{1 / 2}$ and $\epsilon^{2 / 3}$ observed experimentally for incidental dislocation boundaries and geometrically necessary boundaries, respectively.

creases we are led to choose the velocity based on the Peach-Kohler force on this traceless part $v_{a}=D(|\rho|) \times$ $\left(\rho_{\text {ast }}-\delta_{\text {st }} \rho_{a b b} / 3\right) \sigma_{\text {st }}$, making the rate of change of the energy density the negative of a perfect square [18]. (This differs from our earlier glide-only formulation [11].) To ensure that the velocity is proportional to the force per dislocation, we choose $D(|\rho|)=1 /|\rho|=$ $1 / \sqrt{\rho_{i j k} \rho_{i j k} / 2}$. Our theory does not incorporate effects of disorder, dislocation pinning, entanglement, glide planes, crystalline anisotropy, or geometrically unnecessary dislocations. It is designed to provide the simplest framework for understanding dislocation morphologies on this mesoscale.

Our simulations show a close analogy to those of turbulent flows. As in three-dimensional turbulence, defect structures mediate intermittent transfer of morphology to short length scales. (Unlike two-dimensional turbulence, we find no evidence of an inverse cascade-our simulations develop structure only at scales less than or equal to the initial correlation length of the deformation field.) As conjectured [19] for the infinite-Reynolds number Euler equations, our simulations develop singularities in finite time [11]. It is unclear whether our physically motivated equations have weak solutions; our simulations exhibit statistical convergence, but the solutions continue to depend on the lattice cutoff (or on the magnitude of the artificial diffusion added to remove lattice effects) in the continuum limit [18]. Since our simulations exhibit structure down to the smallest scales, we conjecture that this is a kind of sensitive dependence on initial conditions-but here amplified not by passage of time, but by passage through length scales. Since the physical system is cut off by the atomic scale, we may proceed even though our equations are in some sense unrenormalizable in the ultraviolet.

We simulate systems of spatial extent $L$ in two dimensions with periodic boundary conditions; our deformations, rotations, strains, and dislocations are fully threedimensional. The initial plastic distortion field $\beta^{P}$ is a Gaussian random field with decay length $L / 5$ and initial amplitude $\beta_{0}=1$. We apply a second order central upwind scheme designed for Hamilton-Jacobi equations [20] on a finite difference grid. The unstrained simulations presented are at late time, where the elastic energy density is small and smoothly decreasing to zero (see supplementary movies 1 and 2 [21]). The strained simulations in Fig. 4 (see supplementary movie 3 [21]), have uniaxial 
strain in the out-of-plane direction, which is increased by adjusting the external stress $\sigma_{z z}(t)$ to hold $\epsilon(t)$ fixed. The strain rate is $\dot{\epsilon}=0.05 \beta_{0}^{2}$.

Figure 2 shows the orientation-orientation correlation function. Here we see that the cellular (climb-free) structures have nontrivial power-law scaling, but we see nonfractal behavior in the grain boundary morphology allowing climb. In supplementary movie 2 [21], the complex structure of cell walls (climb-free) shows a few primary large-angle boundaries with high dislocation density and many low-angle subboundaries, leading to fuzzy cell walls that are qualitatively different from the grain boundaries (climb and glide, seen in supplementary movie 1 [21]). Table I includes also the correlation function of the total dislocation density; one can show [18], if the elastic strain is zero [22], that $C^{\rho}(\mathbf{r})=-\partial^{2} C_{i i}^{\Lambda}(\mathbf{r})-\partial_{i} \partial_{k} C_{i k}^{\Lambda}(\mathbf{r})$, so $C_{i j}^{\Lambda}(\mathbf{r}) \sim|\mathbf{r}|^{\alpha}$ tells us that $C^{\rho}(\mathbf{r}) \sim|\mathbf{r}|^{\alpha-2}$, implying the exponent relation $\alpha=2-\eta$ in the last column of Table I. The scaling for the correlation function for the total plastic distortion $\beta^{P}$ is not as convincing [18]. Both are consistent with a renormalization-group transformation that rescales the dislocation density by a factor of $b^{-\eta / 2}$ when it rescales the length scale by a factor of $b$. Figure 3 gives a real-space renormalization-group illustration of this self-similarity; the cell walls form a self-similar, hierarchical structure.

Can we reproduce the experimental fractal characterization of cell boundaries? Box counting applied to the dislocation density [as in Fig. 1(a)] gives dimensions that depend strongly on the amplitude cutoff (the dislocation density is self-similar, not a simple fractal). If we first decompose our simulation into cells as in Fig. 4(b), and apply box counting to the resulting cell boundaries, we obtain a fractal dimension of around 1.5 over about a decade [18], compared to the experimental values of 1.64-1.79 [3]. Such a measurement, however, ignores the important variation of wall misorientations with scale (capturing the spatial scaling but missing the amplitude scaling).

Can we reconcile our self-similar cell morphologies with the experimental analyses of Hughes and collaborators [4-6]? Using our boundary-pruning algorithm to identify cell walls, Figs. 4(c) and 4(d) show the cell size and misorientation distributions extracted from an ensemble of initial conditions. The misorientation distribution we find is clearly more scale free (power-law) than that seen experimentally. Under external strain, we do observe the experimental cell structure refinement [Fig. 4(b)], and we find the experimental scaling collapse of the cell-size and misorientation distributions [Figs. 4(c) and 4(d)] and the observed power-law scaling of the mean size and angle with external strain [Figs. 4(e) and 4(f)], albeit with different scaling functions and power laws than those seen in experiments [4-6].

Because we ignore slip systems, spatial anisotropy, and immobile and geometrically unnecessary dislocations, we cannot pretend to reflect real materials. But by distilling these features out of the analysis, we have perhaps elucidated the fundamental differences between cell walls and grain boundaries, and provided a new example of nonequilibrium scale invariance.

We would like to thank S. Limkumnerd, P. Dawson, M. Miller, A. Vladimirsky, R. LeVeque, E. Siggia, and S. Zapperi for helpful and inspiring discussions on plasticity and numerical methods and N. Hansen, D. C. Chrzan, H. Mughrabi, and M. Zaiser for permission of using their TEM micrographs. We were supported by DOE-BES through DE-FG02-07ER46393. Our work was partially supported by the National Center for Supercomputing Applications under MSS090037 and utilized the Lincoln and Abe clusters.

[1] H. Mughrabi, T. Ungar, W. Kienle, and M. Wilkens, Philos. Mag. A 53, 793 (1986).

[2] C. Schwink, Scr. Metall. Mater. 27, 963 (1992).

[3] P. Hähner, K. Bay, and M. Zaiser, Phys. Rev. Lett. 81, 2470 (1998).

[4] D. A. Hughes, Q. Liu, D. C. Chrzan, and N. Hansen, Acta Mater. 45, 105 (1997).

[5] D. A. Hughes, D. C. Chrzan, Q. Liu, and N. Hansen, Phys. Rev. Lett. 81, 4664 (1998).

[6] D. A. Hughes and N. Hansen, Phys. Rev. Lett. 87, 135503 (2001).

[7] M. Miguel, A. Vespignani, S. Zapperi, J. Weiss, and J. Grasso, Nature (London) 410, 667 (2001).

[8] M. Uchic, D. Dimiduk, J. Florando, and W. Nix, Science 305, 986 (2004).

[9] J. Mach, A. Beaudoin, and A. Acharya, J. Mech. Phys. Solids 58, 105 (2010).

[10] A. Roy and A. Acharya, J. Mech. Phys. Solids 53, 143 (2005).

[11] S. Limkumnerd and J.P. Sethna, Phys. Rev. Lett. 96, 095503 (2006).

[12] M. Ortiz, E. Repetto, and L. Stainier, J. Mech. Phys. Solids 48, 2077 (2000).

[13] I. Groma and B. Bakó, Phys. Rev. Lett. 84, 1487 (2000).

[14] W. Pantleon, Acta Mater. 46, 451 (1998).

[15] J.P. Sethna, V. R. Coffman, and E. Demler, Phys. Rev. B 67, 184107 (2003).

[16] J. F. Nye, Acta Metall. 1, 153 (1953).

[17] T. Mura, Micromechanics of Defects in Solids (Martinus Nijhoff Publishers, The Hague, The Netherlands, 1991), 2nd ed.

[18] Y. S. Chen, W. Choi, S. Papanikolaou, and J. P. Sethna (to be published).

[19] A. Pumir and E. D. Siggia, Phys. Fluids A 4, 1472 (1992).

[20] A. Kurganov, S. Noelle, and G. Petrova, SIAM J. Sci. Comput. 23, 707 (2001).

[21] See supplementary material at http://link.aps.org/ supplemental/10.1103/PhysRevLett.105.105501 for four animations showing relaxation with and without climb, evolution under uniaxial loading, and our boundarypruning algorithm.

[22] S. Limkumnerd and J. P. Sethna, Phys. Rev. B 75, 224121 (2007). 